Liver, Pancreas and Biliary Tract

\title{
An a priori prediction model of response to peginterferon plus ribavirin dual therapy in naïve patients with genotype 1 chronic hepatitis C
}

\author{
Angelo Andriulli ${ }^{\mathrm{a}}$, Alessandra Nardi ${ }^{\mathrm{b}}$, Vito Di Marco ${ }^{\mathrm{c}}$, Antonio Massimo Ippolito $^{\mathrm{a}, *}$, \\ Caius Gavrila $^{\mathrm{b}}$, Alessio Aghemo ${ }^{\mathrm{d}}$, Daniele Di Paolo ${ }^{\mathrm{e}}$, Giovanni Squadrito ${ }^{\mathrm{f}}$, \\ Eleonora Grassi ${ }^{\mathrm{d}}$, Vincenza Calvaruso ${ }^{\mathrm{c}}$, Maria Rosa Valvano ${ }^{\mathrm{a}}$, \\ Giuseppina Brancacciog ${ }^{g}$, Antonio Craxi ${ }^{\mathrm{c}}$, Mario Angelico ${ }^{\mathrm{e}}$, Collaborators ${ }^{1}$ \\ a Division of Gastroenterology, Casa Sollievo Sofferenza Hospital, IRCCS, Italy \\ b Department of Mathematics, Tor Vergata University, Roma, Italy \\ c Unit of Gastroenterology, Di.B.I.S., University of Palermo, Italy \\ 'Division of Gastroenterology and Hepatology, Fondazione IRCCS Ca' Granda Ospedale Maggiore Policlinico, Milano, Italy \\ ${ }^{\mathrm{e}}$ Hepatology and Liver Transplantation Unit, University of Tor Vergata, Roma, Italy \\ ${ }^{\mathrm{f}}$ Division of Clinical and Molecular Hepatology, University of Messina, Italy \\ g Clinic of Infectious Diseases, Second University of Napoli, Italy
}

\section{A R T I C L E I N F O}

\section{Article history:}

Received 2 April 2014

Accepted 18 May 2014

Available online 20 June 2014

\section{Keywords:}

Chronic hepatitis

$\mathrm{HCV}$ infection

Peg-interferon and ribavirin treatment

Predictors of sustained virological

response, Rapid virological response

\begin{abstract}
A B S T R A C T
Background: Aim was to select naïve patients with genotype 1 chronic hepatitis $C$ having a high probability of response to Peg-interferon + ribavirin therapy.

Methods: In 1073 patients (derivation cohort), predictors of rapid and sustained virological response were identified by logistic analysis; regression coefficients were used to generate prediction models for sustained virological response. Probabilities at baseline and treatment week 4 were utilized to develop a decision rule to select patients with high likelihood of response. The model was then validated in 423 patients (validation cohort).

Results: In the derivation cohort, 257 achieved rapid virological response and 818 did not, with sustained virological response rates of $80.2 \%$ and $25.4 \%$, respectively; interleukin-28B polymorphisms, fibrosis staging, gamma-glutamyl transferase, and viral load predicted sustained virological response. Assuming a $<30 \%$ sustained virological response probability for not recommending Peg-interferon + ribavirin, 100 patients (25.6\%) in the validation cohort were predicted a priori to fail this regimen. Assuming a $\geq 80 \%$ sustained virological response probability as a threshold to continue with Peg-interferon + ribavirin, 61 patients were predicted to obtain sustained virological response, and 55 of them (90.2\%) eventually did. Conclusions: This model uses easily determined variables for a personalized estimate of the probability of sustained virological response with Peg-interferon + ribavirin, allowing to identify patients who may benefit from conventional therapy.
\end{abstract}

(c) 2014 Editrice Gastroenterologica Italiana S.r.l. Published by Elsevier Ltd. All rights reserved.

\section{Introduction}

For patients with hepatitis $\mathrm{C}$ virus genotype 1 (HCV-1) infection, triple therapy with peg-interferon and ribavirin (PEG/RBV) plus

\footnotetext{
* Corresponding author at: Division of Gastroenterology, Casa Sollievo Sofferenza Hospital, IRCCS, viale Cappuccini 1, San Giovanni Rotondo, Italy.

Tel.: +39 0882 410263; fax: +390882 835411.

E-mail address: antonio.ippolito@me.com (A.M. Ippolito).

1 See Appendix A
}

HCV NS3-NS4a protease inhibitors has boosted sustained virological response (SVR) to levels as high as $63-75 \%[1,2]$. The arrival of novel therapies directed against specific viral targets holds promise of even greater success $[3,4]$. Eligibility for treatment will remain driven largely by efficacy, safety and affordability of antiviral therapies. Conventional dual therapy with PEG/RBV remains efficacious for about $40-50 \%$ of $\mathrm{HCV}-1$ patients who have never been treated before $[5,6]$. Accordingly, the a priori estimation of the individual likelihood of response to PEG/RBV could effectively maximize treatment efficacy while containing health care costs. Indeed, this strategy may drive an individualized treatment algorithm. 
Patients who clear the virus after the initial 4 weeks of PEG/RBV therapy are the best candidates to obtain viral eradication [7]. By pooling data from several trials, we estimated that this condition may be achieved by $25-30 \%$ of naïve HCV-1 patients in Europe and in Australasia [8]. Several guidelines recommend for all previously untreated HCV-1 patients to commence therapy with a 4-week phase of PEG/RBV before the addition of a protease inhibitor [9-12]. Albeit this strategy would allow patients with rapid virological response (RVR) to obviate the use of a protease inhibitor [13] and could eventually be utilized to adjust treatment duration $[5,14]$, it would expose naïve patients unlikely to obtain RVR to suboptimal therapy.

Some host and viral features at baseline that portend a low likelihood of obtaining SVR after PEG/RBV have been recognized [5,14-18]: the staging of liver fibrosis, serum gamma-glutamyl transferase ( $\gamma \mathrm{GT}$ ) levels, HCV viral load, and the interleukin-28B polymorphisms (IL28B) have been concordantly recognized as impacting on the outcome of dual therapy. However, although useful, these predictors are only capable of identifying gross subclasses of patients. Indeed within each subclass, i.e. liver cirrhosis, some patients will and some others will not respond to PEG/RBV. What is currently lacking is a tool to assess the a priori probability of the therapeutic outcome before the patient actually undergoes $\mathrm{PEG} / \mathrm{RBV}$, in the same way as the MELD score estimates the probability of mortality for patients with end-stage liver disease [19].

We aimed to develop a prediction model capable of estimating probability of responding to dual therapy with PEG/RBV at presentation. The robustness of the model was validated in an independent cohort of naïve patients. Such a tool could assist physicians in counselling patients on the most appropriate antiviral therapy.

\section{Materials and methods}

\subsection{Patients}

This investigation refers to a retrospective analysis of a database of 1075 naïve HCV-1 patients with chronic hepatitis, who underwent PEG/RBV treatment at 15 Italian centres between 2005 and 2010. These patients were used as a model derivation cohort. Main characteristics of patients, as well as relevant inclusion and exclusion criteria, have been presented elsewhere [7]. Baseline features recorded included age, gender, serum levels of alanine-aminotransferase (ALT), and gamma-glutamyl transferase ( $\gamma \mathrm{GT}$ ), blood platelet counts, HCV viraemia levels, genotypes of the IL28B locus, diabetes and the stage of liver fibrosis. The latter information was scored from 0 to 4, based on liver histology according to Metavir [20] or liver elastometry (Fibroscan) [21]. Patients with clinically evident cirrhosis, granted by concomitant laboratory abnormalities (such as platelet count $<100,000 \mathrm{~mL}^{-1}$ and/or an APRI score $>1.5$ ) [22] and ultrasound evidence of portal hypertension (portal vein diameter $>13 \mathrm{~mm}$ and spleen longitudinal diameter $(>14 \mathrm{~cm}$ ) [23] we scored as fibrosis stage 4 .

In order to generalize the validity of the prediction model [24], an independent cohort of 423 naïve patients who received PEG/RBV treatment during the same time span was used as an external validation setting. These patients were recruited between 2003 and 2012 at 7 Italian centres who did not participate in the previous study [7].

\subsection{Methods}

Virological analyses and IL28B genotyping were conducted as previously reported [7]. Commercial assays were used to measure HCV RNA levels at local investigational sites, but real-time PCR based methods were used in all centres: levels below the lower limit of quantification at treatment week 4 or at week 24 of follow up off therapy were taken as indicative of RVR or SVR, respectively. All patients had given prior informed consent for use of data and serum for research purposes. The study was conducted in accordance with provisions of the Declaration of Helsinki and Good Clinical Practice Guidelines.

\subsection{Statistical analysis}

Continuous variables, i.e. age (in years), platelet counts $\left(\times 10^{3}\right.$ cells $\left./ \mathrm{mL}\right), \mathrm{HCV}$ RNA levels $(\mathrm{IU} / \mathrm{mL})$, and serum ALT (expressed as times the upper normal limit) were summarized by median, first and third quartile because most of them showed a skew distribution which significantly departed from the normal density. All remaining variables were categorized as follows: diabetes (yes, no), $\gamma \mathrm{GT}$ (normal, abnormal), fibrosis stage (F1, F2, F3, F4), HCV-1 subtype (1a, 1b), HBcAb status (positive, negative), genotype (1a, 1b) and described by absolute and relative frequencies. The genotype of the IL28B locus was given individually as CC, CT and TT. Associations between categorical variables were evaluated by $\chi^{2}$-test; Fisher exact test was preferred in case of sparse tables. Continuous covariates were compared by $t$-test or Wilcoxon rank-sum test when a significant departure from normality was detected. Multivariate analysis was based on the logistic model [24]. The following covariates at baseline were considered as factors potentially affecting the probability of achieving RVR and SVR: age, gender, stage of liver fibrosis, ILB28 genotyping, HBcAb status, diabetes, $\gamma \mathrm{GT}$, platelet count, ALT, HCV viral load, and genotype. Variable selection was based on a non-automated backward selection, taking correlation structure among covariates and clinical interpretation of their effects into account. All numerical data were used as continuous variables in the multivariate analyses, except $\gamma G T$ that was recorded as normal $v s$ abnormal levels. Since the distribution of HCV RNA levels was extremely skew, the $\log _{10}$ transformation of values was used. Of considered variables, the maximum percentage of missing data was $3 \%$ for ALT determination. No attempt was made to impute missing data, and incomplete observations were excluded from multivariable analysis. In the final logistic models only four patients had incomplete information on selected covariates. When a model to predict SVR was developed, in addition to baseline features, obtainment of RVR was also considered as an on-treatment predictor.

To evaluate the ability of the proposed models to predict RVR and SVR, receiver operating characteristic (ROC) curves were computed, and the area under the curve (c-statistic) reported. The Hosmer and Lemeshow test was performed to detect possible lack of fit of the final models to the observed data [25]. The confidence interval displacement diagnostic was used to measure the influence of individual observations on the regression estimates [26]. The change in deviance and in $\chi^{2}$ statistic attributable to deleting the individual observation was considered as well. Poorly fitted observations were identified by the standardized deviance and Pearson residuals. All analyses were performed using SAS version 9.3.

\section{Results}

\subsection{Model derivation cohort}

The cohort included 414 (38.5\%) subjects who obtained SVR and 661 (61.5\%) who did not. Baseline characteristics in the two subgroups of patients are shown in Table 1. SVR patients were younger, had lower baseline viral load, more frequently had genotype CC of the IL28B polymorphism, and included fewer cirrhotics. In addition, they presented more frequently with normal $\gamma \mathrm{GT}$ levels, and were less often diabetics. After treatment with PEG/RBV, 257 
Table 1

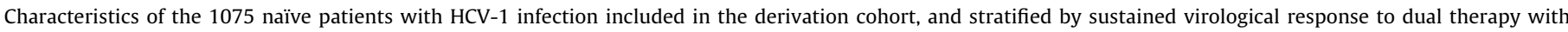
peg-interferon and ribavirin.

\begin{tabular}{|c|c|c|c|c|c|c|}
\hline & \multicolumn{2}{|l|}{$\operatorname{SVR}(n=414,38.51 \%)$} & \multicolumn{2}{|c|}{ No SVR $(n=661,61.49 \%)$} & \multirow[t]{2}{*}{$p$ value } & \multirow[t]{2}{*}{ Missing } \\
\hline & Median or number & Q1-Q3 or \% & Median or number & Q1-Q3 or $\%$ & & \\
\hline Age (years) & 52 & $43-62$ & 57 & $48-64$ & $<0.0001$ & 0 \\
\hline Gender (male) & 230 & 55.56 & 393 & 59.46 & 0.2075 & 0 \\
\hline Fibrosis & & & & & $<0.0001$ & 0 \\
\hline F1 & 140 & 33.82 & 141 & 21.33 & & \\
\hline $\mathrm{F} 2$ & 111 & 26.81 & 160 & 24.21 & & \\
\hline F3 & 71 & 17.15 & 118 & 17.85 & & \\
\hline $\mathrm{F} 4$ & 92 & 22.22 & 242 & 36.61 & & \\
\hline IL28B & & & & & $<0.0001$ & 0 \\
\hline CC & 183 & 44.20 & 103 & 15.58 & & \\
\hline $\mathrm{TT} / \mathrm{CT}$ & 231 & 55.80 & 558 & 84.42 & & \\
\hline Genotype (1a) & 59 & 14.53 & 71 & 10.99 & 0.0893 & 23 \\
\hline HBcAb status (positive) & 91 & 22.25 & 151 & 23.23 & 0.7111 & 16 \\
\hline Diabetes (yes) & 36 & 8.70 & 94 & 14.22 & 0.0069 & 0 \\
\hline$\gamma \mathrm{GT}$ (abnormal) & 152 & 36.71 & 393 & 59.64 & $<0.0001$ & 2 \\
\hline Platelets $\left(\times 10^{3} / \mathrm{mL}\right)$ & 200 & $159-229$ & 180 & $123-223$ & $<0.0001$ & 0 \\
\hline $\operatorname{ALT}(\times \mathrm{ULN})^{\mathrm{a}}$ & 2.0 & $1.2-3.0$ & 2.0 & $1.5-3.0$ & 0.0288 & 16 \\
\hline HCV RNA $\left(\times 10^{3} \mathrm{IU} / \mathrm{mL}\right)$ & 503 & $160-1330$ & 909 & $386-2099$ & $<0.0001$ & 0 \\
\hline Rapid virological response & 206 & 49.76 & 51 & 7.72 & $<0.0001$ & 0 \\
\hline
\end{tabular}

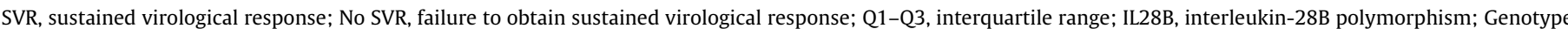

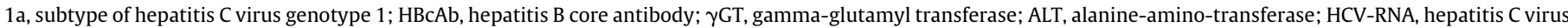
RNA serum levels.

a ULN, upper limit of normal.

subjects (23.9\%) achieved RVR and 818 did not; SVR rates were $80.2 \%$ and $25.4 \%$, respectively.

\subsection{Development of logistic models to predict virological outcomes}

\subsubsection{RVR prediction (logistic Model A)}

Among baseline features, IL28B genotype, staging of liver fibrosis, $\gamma$ GT levels, and viral load were identified as predictors of RVR. As RVR rates did not significantly differ among patients with F1, F2, and F3 fibrosis, in the final logistic model fibrosis was categorized as $<\mathrm{F} 4$ vs F4 (Model A, Table 2). The area under the cross-validated ROC curve was 0.745 (Fig. 1A). Two individual patients were found to be highly influential on the fitting of the final model. In order to test the model robustness, they were excluded from the analysis, and the model was refitted: the maximum change in parameter estimates was observed for the variable fibrosis, whose regression coefficient decreased from -0.76 to -0.71 value; all the selected variables remained highly significant. Inspection of residuals led to identify 34 poorly predicted observations (3.2\%): in all, but one of them, RVR was obtained, although the predicted probability was lower than 0.14. The regression coefficients, shown in Table 2 Panel A, were used to develop an RVR score for each individual patient according to the following formula:

$$
\begin{aligned}
\text { RVR score }= & 4.21 \text { (theintercept })-(1.37 \text { ifIL28BTT } / C T) \\
& -(0.63 \text { ifF4fibrosis })-(0.47 \text { ifabnormalgGT }) \\
& -(0.71 \times 1 \log 10 \text { HCVRNA }) .
\end{aligned}
$$

According to the logistic model, the a priori estimated probability for the patient to obtain RVR was derived by the equation:

ProbabilityofRVR $=\frac{\operatorname{Exp}(R V R \text { Score })}{1+\operatorname{Exp}(\text { RVRScore })}$.

\subsubsection{SVR prediction given RVR status (logistic Model B)}

At multivariate analysis (Table 2, Panel B), predictors of SVR included age and RVR, in addition to the IL28B genotype, fibrosis stage, yGT levels, and baseline viral load. Again, no difference was observed in the effect of F1, F2 and F3 on the rate of SVR, thus fibrosis stage was categorized as $<\mathrm{F} 4$ vs F4. A significant interaction between RVR and fibrosis emerged from the analysis. Although only $55(16.5 \%$ ) cirrhotics experienced RVR, they had a much higher probability to obtain SVR than those who did not achieve RVR $(\mathrm{OR}=30.0)$. Conversely, for non-cirrhotic patients, achievement of RVR had a considerably lower impact in predicting SVR $(O R=5.07)$. Conversely, fibrosis F4 was a strong predictor of failure to respond to PEG/RBV only in the absence of RVR. As illustrated in Fig. 2a, the predictive capability of Model $B$, as measured by the area under the cross-validated ROC curve, was 0.818 . Five observations were highly influent in determining the final parameter estimates: all these patients obtained RVR despite having a degree of fibrosis F4 but none of them achieved SVR. When the model was re-fitted after excluding these individuals, the major change was observed in the interaction coefficient which increased from 1.77 to 3.20. All the selected covariates remained highly significant. Residual analysis identified 29 outliers (2.7\%): 19 of them achieved SVR despite not experiencing RVR and having a $<0.14$ predicted probability of SVR. The remaining 10 patients failed to clear the infection despite their estimated probability was higher than 0.87 . We derived a SVR predictive score according to the parameter estimates (i.e., intercept value and regression coefficients) given in Table 3, by the following formula:

$$
\begin{aligned}
\text { SVRScore }= & 3.95-(0.02 \times \text { age })-(1.22 \text { ifIL28BTT } / C T) \\
& -(0.55 \text { ifabnormalgGT })-(0.44 \times \log 10 \text { HCVRNA }) \\
& +(1.62 \text { ifRVR })-(0.80 \text { ifFibrosis }=\text { F4 }) \\
& +(1.78 \text { ifRVRandFibrosis }=\text { F4 }) .
\end{aligned}
$$

Finally, the probability of SVR was derived as follows:

Probability of SVR $=\frac{\operatorname{Exp}(\text { SVR Score })}{1+\operatorname{Exp}(\text { SVRScore })}$.

\subsubsection{Estimate of the a priori probability to achieve SVR after} Peg-IFN and ribavirin

After having estimated the probability of RVR by Model A and the probability of SVR given RVR status by Model B, we estimated 
Table 2

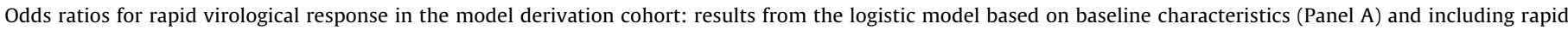
virological response at 4 weeks (Panel B).

\begin{tabular}{|c|c|c|c|c|c|}
\hline \multicolumn{6}{|l|}{ Panel A } \\
\hline Variable & Parameter estimate & Odds ratio & \multicolumn{2}{|c|}{ 95\% Wald confidence interval } & $p$ value \\
\hline Intercept & 4.21 & & & & \\
\hline IL28B TT/TC vs CC & 1.37 & 0.25 & 0.19 & 0.35 & $<0.0001$ \\
\hline Fibrosis (stage F4 vs stage $<\mathrm{F} 4$ ) & -0.63 & 0.53 & 0.37 & 0.77 & 0.0007 \\
\hline$\gamma \mathrm{GT}$ (abnormal vs normal) & -0.47 & 0.63 & 0.46 & 0.86 & 0.0037 \\
\hline HCV RNA ( $\times$ unit, $\log _{10}$ scale) & -0.71 & 0.49 & 0.40 & 0.61 & $<0.0001$ \\
\hline \multicolumn{6}{|l|}{ Panel B } \\
\hline Variable & Parameter estimate & Odds ratio & \multicolumn{2}{|c|}{ 95\% Wald confidence interval } & $p$ value \\
\hline Intercept & 3.96 & & & & \\
\hline Age (years) & -0.02 & 0.83 ( 10 years $)$ & 0.73 & 0.94 & 0.0029 \\
\hline IL28B TT/CT vs CC & -1.22 & 0.30 & 0.21 & 0.42 & $<0.0001$ \\
\hline$\gamma \mathrm{GT}$ (abnormal vs normal) & -0.55 & 0.58 & 0.43 & 0.78 & 0.0004 \\
\hline HCV RNA ( $\times$ unit, $\log _{10}$ scale) & -0.44 & 0.65 & 0.52 & 0.81 & $<0.0001$ \\
\hline Fibrosis (F4 vs <F4) & -0.80 & & & & 0.0001 \\
\hline RVR (yes vs no) & 1.62 & & & & $<0.0001$ \\
\hline RVR*Fibrosis & 1.78 & & & & 0.0003 \\
\hline RVR yes $v s$ no if Fibrosis < F4 & & 5.07 & 3.37 & 7.63 & \\
\hline RVR yes $v s$ no if Fibrosis F4 & & 29.95 & 12.62 & 71.06 & \\
\hline Fibrosis F4 vs <F4 if RVR yes & & 2.67 & 1.11 & 6.40 & \\
\hline Fibrosis F4 vs <F4 if RVR no & & 0.45 & 0.30 & 0.68 & \\
\hline
\end{tabular}

Total number of patients $=1073$ (missing 2); patients with sustained virological response $(\mathrm{SVR})=414$ with rapid virological response $(\mathrm{RVR}=257$ ).

IL28B, interleukin-28B polymorphism; $\gamma \mathrm{GT}$, gamma-glutamyl transferase; HCV-RNA, hepatitis C virus RNA serum levels; RVR, rapid virological response.

the a priori probability for patients to achieve an SVR when PEG/RBV would be administered. Indeed, Model B actually allows computing two different SVR probabilities: the first one in the event a patient obtains RVR, the second one in the event he/she does not. Since at presentation it is unknown whether a patient will or will not obtain RVR, an a priori estimate of SVR will necessarily result from a weighted balance of two distinct SVR probabilities. A basic rule of probability allows to compute the a priori probability of SVR by means of the following equation:

$$
\begin{aligned}
\text { ProbabilityofSVR }= & (\text { ProbabilityofSVRifRVR }) \times(\text { ProbabilityofRVR }) \\
& +(\text { ProbabilityofSVRwithoutRVR }) \\
& \times(\text { ProbabilityofnoRVR }) .
\end{aligned}
$$

Table 3

\begin{tabular}{|c|c|c|c|c|c|c|c|}
\hline \multicolumn{8}{|l|}{ (A) } \\
\hline \multirow[t]{2}{*}{ Probability cut-off level } & \multirow{2}{*}{\multicolumn{2}{|c|}{ Selected patients (out of 1073) }} & \multicolumn{5}{|c|}{ No SVR $(n=659)$} \\
\hline & & & \multicolumn{2}{|c|}{ Correctly classified } & \multicolumn{2}{|c|}{ Incorrectly classified } & False negative rate \\
\hline 0.20 & $222(20.7 \%)$ & & 188 & & 34 & & 15.3 \\
\hline 0.25 & $364(33.9 \%)$ & & 301 & & 62 & & 17.0 \\
\hline 0.30 & $469(43.7 \%)$ & & 369 & & 100 & & 21.3 \\
\hline 0.35 & $573(53.4 \%)$ & & 447 & & 126 & & 22.0 \\
\hline 0.40 & $662(61.7 \%)$ & & 504 & & 158 & & 23.9 \\
\hline 0.45 & $734(68.4 \%)$ & & 546 & & 188 & & 25.6 \\
\hline 0.50 & $776(72.3 \%)$ & & 560 & & 216 & & 27.8 \\
\hline 0.55 & $834(77.7 \%)$ & & 590 & & 244 & & 29.3 \\
\hline 0.60 & $877(81.7 \%)$ & & 610 & & 267 & & 30.4 \\
\hline \multicolumn{8}{|l|}{ (B) } \\
\hline \multirow[t]{2}{*}{ Probability level } & \multirow[t]{2}{*}{ Selected patients $(n=1073)$} & \multicolumn{4}{|c|}{$\operatorname{SVR}(n=414)$} & Specificity & False positive rate \\
\hline & & \multicolumn{2}{|c|}{ Correctly classified } & \multicolumn{2}{|c|}{$\overline{\text { Incorrectly classified }}$} & & \\
\hline 0.60 & $263(24.5 \%)$ & 217 & & 46 & & 93.0 & 17.5 \\
\hline 0.65 & $235(21.9 \%)$ & 197 & & 38 & & 94.2 & 16.2 \\
\hline 0.70 & $209(19.5 \%)$ & 182 & & 27 & & 95.9 & 12.9 \\
\hline 0.75 & $185(17.2 \%)$ & 162 & & 23 & & 96.5 & 12.4 \\
\hline 0.80 & $150(14.0 \%)$ & 134 & & 16 & & 97.6 & 10.7 \\
\hline 0.85 & $116(10.8 \%)$ & 105 & & 11 & & 98.3 & 9.5 \\
\hline 0.90 & $65(6.1 \%)$ & 59 & & 6 & & 99.1 & 9.2 \\
\hline
\end{tabular}

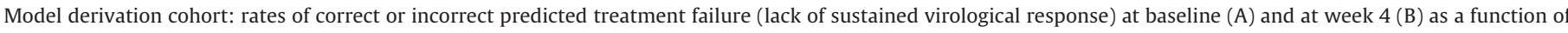
increasing probability cut-off values.

SVR, sustained virological response. 

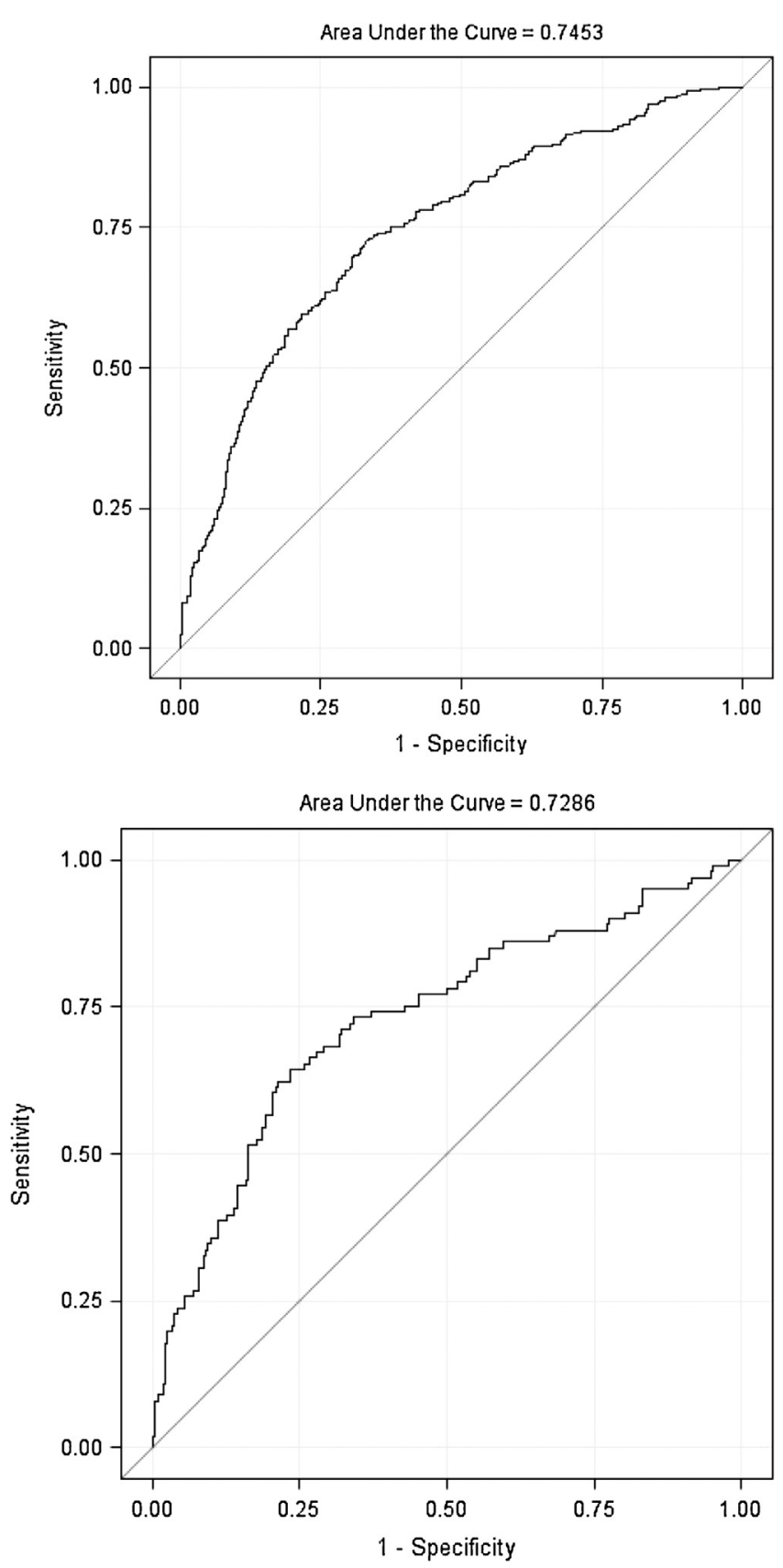

Fig. 1. Cross-validated receiver operating characteristic curve for the prediction of rapid virological response (Model A) in the derivation cohort (Panel a) and in the validation cohort (Panel b).

Of note, in this weighted average weights are given the probabilities of RVR, estimated according to model A.

Fig. 3 shows the relationship between the estimated probability of SVR at presentation (Fig. 3a) and that at week 4 (Fig. 3b), together with the predicted RVR at presentation. As shown in Fig. 3a, the $a$ priori estimated probability of SVR was constantly higher that the probability of RVR. Fig. 3b highlights how knowing the RVR status significantly modified this relationship.

\subsubsection{Development of a two-step predictive algorithm}

Individual estimated probabilities of SVR, both at presentation and at week 4, can be used to select patients who might reliably benefit from conventional dual therapy with PEG/RBV. The proposed procedure includes two sequential steps.
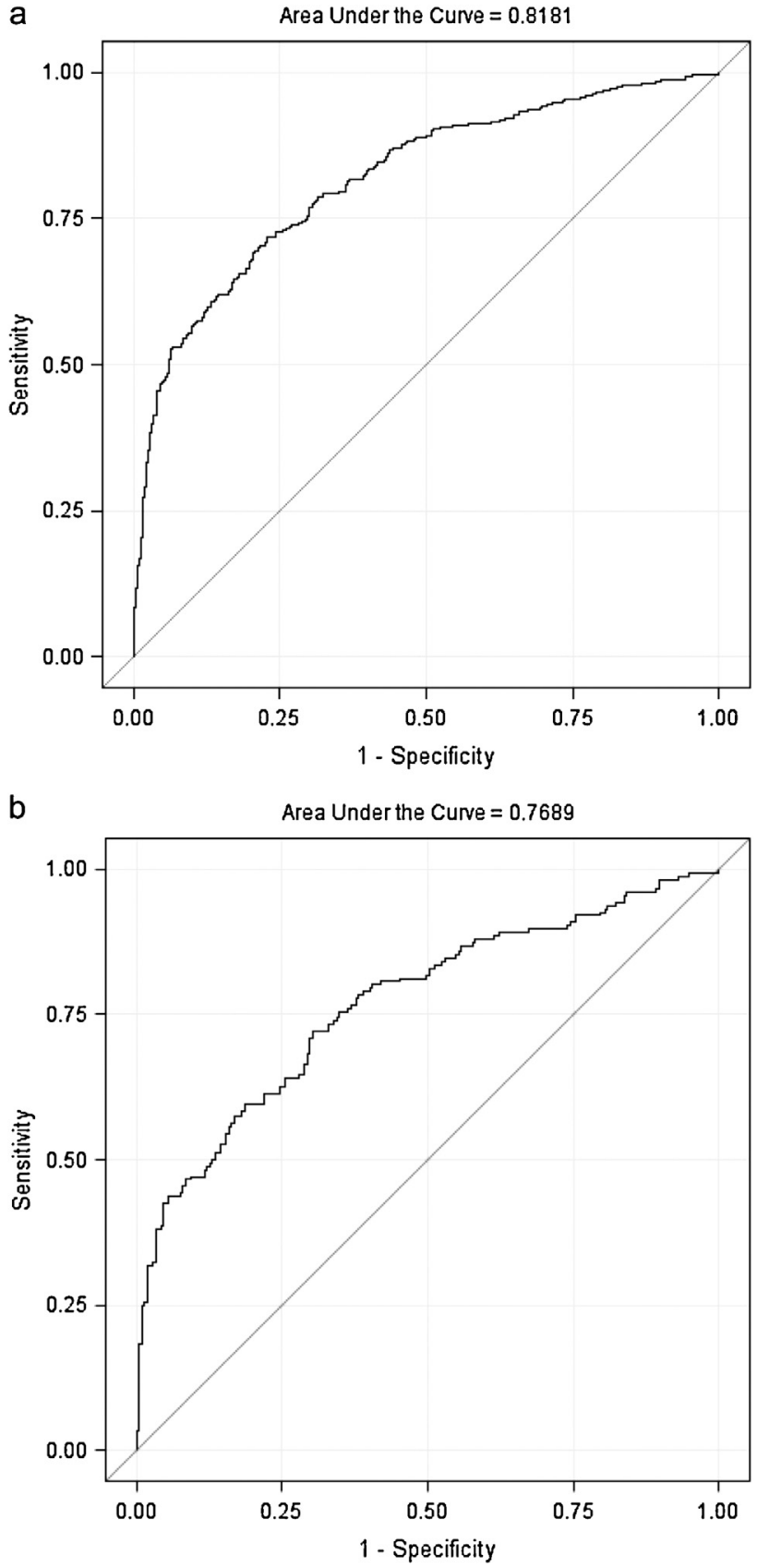

Fig. 2. Cross-validated receiver operating characteristic curve for sustained virological response, given the rapid virological response status (Model B) in the derivation cohort (Panel a), and in the validation cohort (Panel b). RVR, rapid virological response

Step 1: At presentation, the individual probability of SVR is computed according to formula (3). As an exemplification, we consider a hypothetical 60-year-old patient with IL28B CC genotype, stage 4 fibrosis, normal serum $\gamma \mathrm{GT}$, and a HCV RNA level of $350,000 \mathrm{IU} / \mathrm{L}$. Based on Model A, the $a$ priori probability of RVR would be 0.41 (formula (1)). By applying Model B, this patient is estimated to have an SVR probability of 0.95 in the event of RVR (formula (2)), and of 0.38 in the event of no RVR. In this patient with favourable characteristics but stage 4 of fibrosis, it seems worthwhile to remark that knowledge of the RVR status substantially modifies his/her probability to obtain an SVR. However, since at presentation the RVR status is unknown, the a priori probability of SVR is computed by combining these two probabilities 

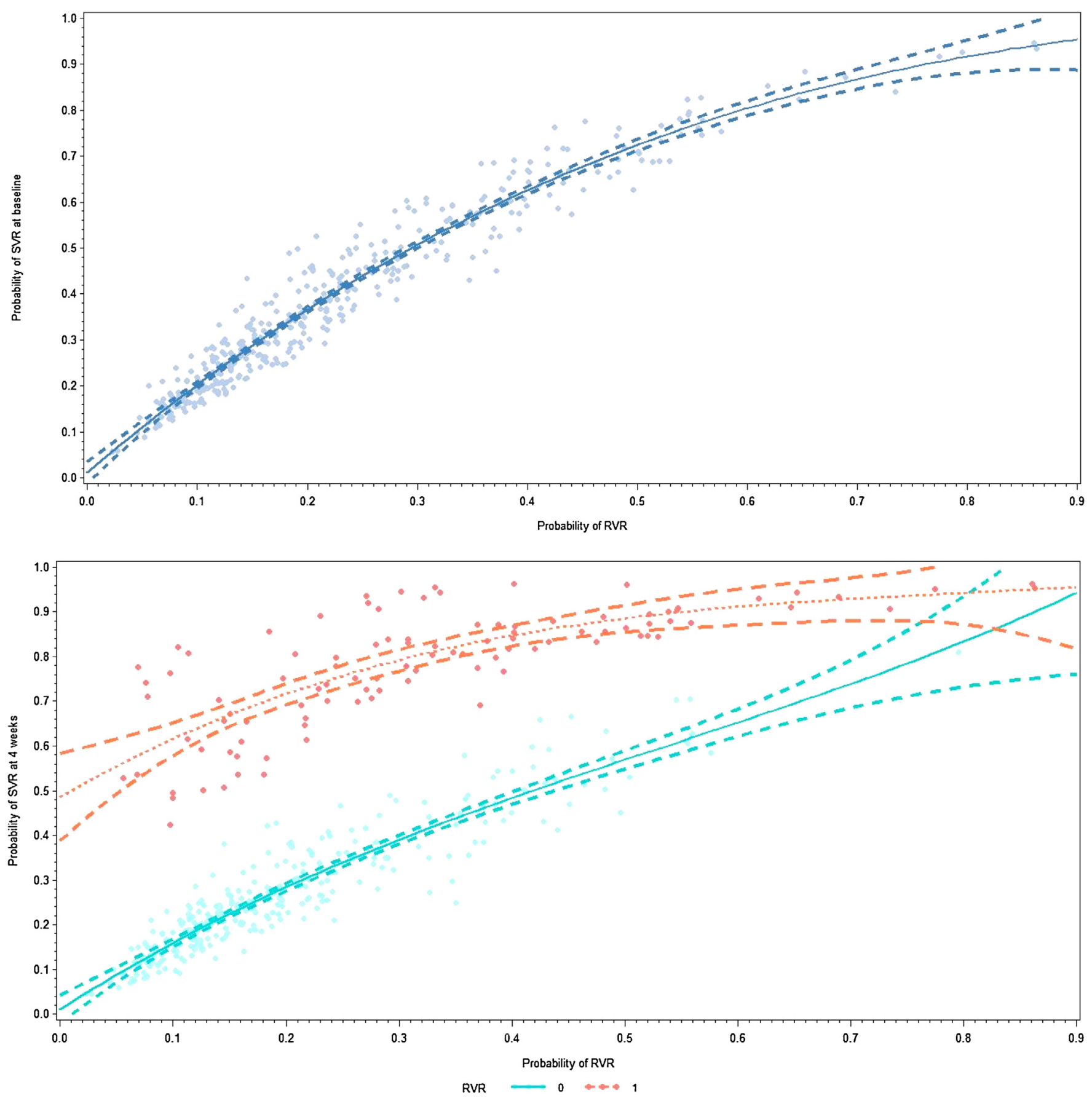

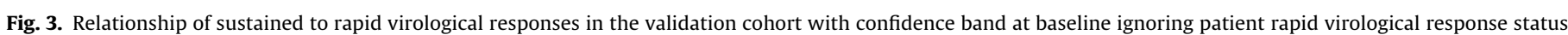
(Panel a) and at 4 weeks given patient rapid virological response status (Panel b). RVR, rapid virological response

according to formula (3), and is equal to 0.61. Based on these individual a priori SVR probabilities, we can identify patients unlikely to respond to PEG/RBV (futile treatment). Table 3A illustrates different thresholds to be used to define a treatment futile, together with the corresponding false negative estimated rates.

Step 2: Patients having an a priori probability higher than the chosen futility threshold will be started on PEG/RBV and reevaluated at treatment week 4 . This would be the case for our hypothetical patient who was computed with a futility SVR threshold of 0.60 . The probability of SVR can now be re-evaluated according to model B (formula (2)), using the actual RVR status: in the event the patient obtained an
RVR, his probability of achieving SVR would increase from 0.61 (estimated at presentation) to 0.95 . The model now allows to identify patients who may continue PEG/RBV and be expected to obtain SVR with a high degree of probability. Different SVR thresholds are considered in Table 3B, together with the corresponding estimated false positive rates.

\subsection{Validation cohort}

The validation cohort comprised 423 naïve patients who received $\mathrm{PEG} / \mathrm{RBV}$ treatment, and included 187 patients (44.2\%) who obtained SVR. Baseline characteristics of patients in this cohort 
are shown in Supplementary Table S1. These patients were significantly younger, had less severe liver fibrosis, were more often IL28B genotype CC, and had lower viral load and $\gamma \mathrm{GT}$ levels. Despite these differential features, the performance of the RVR and SVR prediction models remained robust: $c$-statistics were 0.7286 and 0.7689 , respectively.

\section{Discussion}

In the rapidly evolving era of anti-HCV drug development, the use of new drugs will conceivably largely depend on their cost and their affordability by the healthcare systems, which may vary from country to country. In this perspective, it is important to be able to distinguish between patients who must be treated with new drugs, and those who can be cured with conventional dual therapy (PEG/RBV). The recently issued EASL Clinical Practice Guidelines on the management of hepatitis $C$ virus infection yet support a potential role for dual therapy for selected treatment-naïve patients with genotype 1 infection [27].

In HCV-1 patients several host and viral features are used to optimize response to conventional therapy with PEG/RBV [5,16-18]. However, these predictors are only able to delineate broad categories of patients with low or high chances of treatment success, but are unable to personalize treatment outcome. Indeed, it is common knowledge that within each of these categories, i.e. advanced/severe liver fibrosis or different IL28B genotype patterns, some patients will and some others will not respond to PEG/RBV. It follows that the likelihood of response depends not only on the identified factors, but also on their interplay with other variables.

The present study demonstrates the accuracy of a new model for identifying patients with high or low probabilities of response to PEG/RBV therapy. In contrast to previous studies that reported percentages of SVR associated with each predictor, we cumulated all data to generate a two-step logistic model for a personalized estimate of an a priori probability of SVR. The model computation incorporates five baseline variables (interleukin-28B polymorphisms, age, liver fibrosis, serum $\gamma$ GT levels, and HCV viral load) and a single on-treatment viral kinetic, i.e. the RVR. Due to the strong relationship between RVR and SVR, the a priori probability of SVR was computed as the weighted average of the estimated SVR probabilities in the presence or absence of RVR.

Our modelling proved to be highly accurate in predicting either a negative or a positive outcome after dual PEG/RBV therapy, both in the derivation and the validation cohort. The performance of the final model B showed a $c$-statistic of 0.818 in the derivation cohort, and of 0.769 in the validation setting (Fig. 2A and B, respectively).

A practical application of this two-step predictive model in the clinical setting is described for the validation cohort in Supplementary Fig. S1. Assuming an a priori probability of SVR of 0.30 as a plausible futile threshold to administer conventional PEG/RBV dual therapy, our model identified 100 (25.6\%) of 391 patients (with complete data) predicted to fall below this limit. The actual data showed that, when these patients were challenged with PEG/RBV, 76 of them did not respond, i.e. were correctly predicted. As a practical consequence, these patients should be immediately considered for alternative treatment. All the remaining 291 patients with an a priori probability of SVR $\geq 30 \%$ were re-evaluated after 4 weeks of PEG/RBV, at a time when it became evident that they had, or had not, obtained RVR. Assuming now a cut-off of $\geq 80 \%$ as a plausible SVR rate to recommend continuation of PEG/RBV treatment, the model identified 61 patients $(15.6 \%$ of the whole cohort). The actual data showed that 55 (90.2\%) of them achieved SVR, i.e. were correctly predicted. As illustrated in the algorithm shown in Supplementary Fig. S1 our prediction model identified 230 patients who, after re-evaluation at week 4 of PEG/RBV therapy, showed a $<80 \%$ probability of obtaining SVR. Notably, this subgroup of patients includes also 27 patients who achieved RVR but not SVR.

An additional finding of this study was that achieving RVR appears to be crucial in cirrhotic patients, substantially increasing their probability of obtaining SVR [28,29]. This observation reinforces the validity of our predictive model which is comprehensive of baseline as well as on-treatment features.

While we are aware that the validity of the above proposed strategy for allocating treatment may require a further prospective validation, we feel that the information provided by our modelling will retain value even in the coming era of new treatment regimens $[3,4,26]$ for several reasons. First, even though guidelines will gravitate towards these newer preferred treatments, the universal use of present or future triple therapies is certainly an overtreatment for the subset of patients with favourable clinical, genetic, and virological profiles $[12,13,30]$. Several scientific societies issued position papers stating that treatment-naïve patients with favourable baseline and on-treatment predictive features do not necessarily deserve triple therapy $[11,12]$, a recommendation which has undergone a formal validation in a recent clinical trial [13]. Secondly, facing the cost of upcoming interferon-free therapies will be an issue. In many parts of the world, including those with more extensive resources such as the United States and some European nations, the cost of drugs will likely determine a disparity in access to care with some regimens being prohibitive in countries with budgetary and resource constraints [31]. Aronsohn and Jensen have recently pointed to the issue of distributive justice with the arrival of new antivirals, as this medical breakthrough will be coupled with resource scarcity [32]. This debate is conventionally approached by the argument that "easy to treat" HCV-1 patients might have access to double therapy only, the more costly new therapies being restricted to patients with "difficult to treat" infections [33].

A major limitation of the present study is that this model deserves further external validation in an independent dataset of non-Italian patients of either Caucasian and non-Caucasian ethnic background. Finally, its prediction power for non HCV-1 genotypes, and in particular for genotype 3 , should also be assessed.

In conclusion, the proposed model, which uses easily determined and objective variables, allows to identify naïve $\mathrm{HCV}-1$ patients with a high probability of failure to respond to PEG/RBV therapy, as well as those with a high probability of obtaining SVR with the conventional dual regimen. In doing so it provides useful additional information for clinicians to aid their therapeutic decision-making process.

\section{Conflict of interest}

None declared.

\section{Appendix A. Collaborators}

Giovanni Raimondo (Division of Clinical and Molecular Hepatology, University of Messina, Italy), Michele Milella (Clinic of Infectious Diseases, University of Bari, Italy), Filomena Morisco (Division of Gastroenterology, Department of Clinical Medicine and Surgery, Federico II University of Napoli, Italy), Giovanna Fattovich (Clinic of Gastroenterology, Department of Medicine, University of Verona, Italy), Martina Felder (Division of Gastroenterology, Central Hospital, Bolzano, Italy), Antonina Smedile (Department of Medical Sciences, University of Torino and Department of Gastroenterology and Hepatology, Azienda Ospedaliera Città della Salute e della Scienza, Torino, Italy), Massimo Fasano (Clinic of Infectious Diseases, University of Foggia, Italy), Pietro Gatti (DIMO Medical Oncology, University of Bari, Italy), Nicola Andriulli (Department of Pharmaceutical Chemistry, La Sapienza 
University, Roma, Italy), Paolo Tundo (Division of Infectious Diseases, S. Caterina Novella Hospital, Galatina, Italy), Michele Barone (Clinic of Gastroenterology, University of Bari, Italy), Raffaele Cozzolongo (Division of Gastroenterology, "S. de Bellis" Hospital, IRCCS, Castellana Grotte, Italy), Giovanna D'Andrea (Department of Clinical and Experimental Medicine, University of Foggia, Italy), Giuseppe Mazzella (Clinic of Gastroenterology, University of Bologna, Italy), Teresa Santantonio (Clinic of Infectious Diseases, University of Foggia, Italy),

\section{Appendix B. Supplementary data}

Supplementary material related to this article can be found, in the online version, at http://dx.doi.org/10.1016/j.dld.2014.05.015.

\section{References}

[1] Poordad F, McCone J, Bacon BR, et al. Boceprevir for untreated chronic HCV genotype 1 infection. New England Journal of Medicine 2011:364:1195-206.

[2] Jacobson IM, McHutchison JG, Dusheiko G, et al. Telaprevir for previously untreated chronic hepatitis C virus infection. New England Journal of Medicine 2011;364:2405-16.

[3] Welsch C, Jesudian A, Zeuzem S, et al. New direct-acting antiviral agents for the treatment of hepatitis C infection and perspectives. Gut 2012;61(Suppl. 1):i36-46.

[4] Schaefer E, Chung R. Anti hepatitis C virus drugs in development. Gastroenterology 2012;142:1340-50

[5] Mangia A, Minerva N, Bacca D, et al. Individualized treatment duration for hepatitis $C$ genotype 1 patients: a randomized controlled trial. Hepatology 2008:47:43-50

[6] Chou R, Hartung D, Rahman B, et al. Comparative effectiveness of antiviral treatment for hepatitis $C$ virus infection in adults: a systematic review. Annals of Internal Medicine 2013;158:114-23.

[7] Andriulli A, Di Marco V, Margaglione M, et al. Identification of naïve HCV-1 patients with chronic hepatitis who may benefit from dual therapy with peg-interferon and ribavirin. Journal of Hepatology 2014;60:16-21.

[8] Andriulli A, Iacobellis A, Valvano MR, et al. Estimates of HCV-1 patients obtaining RVR following dual therapy with peg-interferon and ribavirin. Digestive Diseases and Sciences 2013;58:1371-82.

[9] Ghany MG, Nelson DR, Strader DB, et al. An update on treatment of genotype 1 chronic hepatitis $C$ virus infection: 2011 practice guidelines by the American Association for the Study of the Liver. Hepatology 2011;54:1433-44.

[10] Yee HS, Ghang ME, Pocha Ch, et al. Update on the management and treatment of hepatitis C virus infection: recommendations from the Department of Veterans Affairs Hepatitis C Resource Center Program and the National Hepatitis C Program Office. American Journal of Gastroenterology 2012;107:669-89.

[11] European Association for the Study of the Liver. EASL Practice Guidelines: management of hepatitis C virus infection. Journal of Hepatology 2011;55:245-64.

[12] Coco B, Caraceni P, Aghemo A, et al. Triple therapy with first-generation protease inhibitors for patients with genotype 1 chronic hepatitis C: recommendations of the Italian Association for the Study of the Liver (AISF). Digestive and Liver Disease 2014;46:18-24.
[13] Pearlman BL, Ehleben C. Hepatitis C genotype 1 virus with low viral load and rapid virologic response to peginterferon/ribavirin obviates a protease inhibitor. Hepatology 2014;59:71-7.

[14] Zeuzem S, Buti M, Ferenci P, et al. Efficacy of 24 weeks treatment with peginterferon alfa-2b plus ribavirin in patients with chronic hepatitis $C$ infected with genotype 1 and low pretreatment viremia. Journal of Hepatology 2006; 44:97-103.

[15] Zeuzem S, Rodriguez-Torres M, Reddy KR, et al. Optimized threshold for serum HCV RNA to predict treatment outcomes in hepatitis $C$ patients receiving peg-interferon alfa-2a/ribavirin. Journal of Viral Hepatitis 2012;19:766-74.

[16] Bruno S, Vierling JM, Esteban R, et al. Efficacy and safety of peg-interferon alfa-2a (40 KD) plus ribavirin in hepatitis $C$ patients with advanced fibrosis and cirrhosis. Hepatology 2010;51:388-97.

[17] Petta S, Macaluso FS, Barcellona MR, et al. Serum $\gamma$-glutamyl transferase levels, insulin resistance and liver fibrosis in patients with chronic liver diseases. PLoS ONE 2012;7:e51165.

[18] Thompson AJ, Muir AJ, Sulkowski MS, et al. Interleukin-28B polymorphism improves viral kinetics and is the strongest pretreatment predictor of sustained virologic response inn genotype 1 hepatitis $C$ virus. Gastroenterology 2010;139:120-9.

[19] Malinchoc M, Kamath PS, Gordon FD, et al. A model to predict poor survival in patients undergoing transjugular intrahepatic portosystemic shunts. Hepatology 2000;31:864-71.

[20] Bedossa P, Poynard T. An algorithm for the grading of activity in chronic hepatitis C. The METAVIR cooperative study group. Hepatology 1996:24:289-93.

[21] Castera L. Non invasive method to assess liver disease in patients with hepatitis B and C. Gastroenterology 2012;142:1293-302.

[22] Iacobellis A, Mangia A, Leandro G, et al. External validation of biochemica indices for non-invasive evaluation of liver fibrosis in HCV chronic hepatitis. American Journal of Gastroenterology 2005;100:868-73.

[23] Symonosky V. The diagnosis of cirrhosis by high-resolution ultrasound of the liver surface. British Journal of Radiology 1999;72:29-34.

[24] Altman D. Prognosis and prognostic research: validating a prognostic model. BMJ 2009;338:1432-5.

[25] Hosmer DW, Lemershow S. A goodness-of-fit test for the multiple logistic regression model. Communications in Statistic 1980;10:1043-69.

[26] Pregibon D. Logistic regression diagnostics. Annals of Statistics 1981;9:705-24.

[27] EASL Clinical Practice Guidelines: management of hepatitis C virus infection. Journal of Hepatology 2014;60:392-420.

[28] Aghemo A, Degasperi E, Rumi MG, et al. Cirrhosis and rapid virological response to peginterferon plus ribavirin determine treatment outcome in HCV-1 IL28B rs1297860 CC patients. BioMed Research International 2013;2013:580796.

[29] Di Marco V, Calvaruso V, Grimaudo S, et al. Role of IL-28B and inosine triphospgatase polymorphisms in efficacy and safety of peg-interferon and ribavirin in chronic hepatitis C compensated cirrhosis with and without oesophageal varices. Journal of Viral Hepatitis 2013:20:113-21.

[30] Aghemo A, De Francesco R. New horizons in hepatitis C antiviral therapy with direct-acting antivirals. Hepatology 2013;58:428-38.

[31] Di Marco V, Covolo L, Calvaruso V, et al. Who is more likely to respond to dua treatment with pegylated-interferon and ribavirin for chronic hepatitis C? A gender-oriented analysis. Journal of Viral Hepatitis 2013;20:790-800.

[32] Aronsohn A, Jensen D. Distributive justice and the arrival of direct-acting antivirals: who should be first in line? Hepatology 2011;53:1789-91.

[33] Alberti A, Colombo M, Craxi A, et al. The dilemma for patients with chronic hepatitis C: treat now or warehouse? Digestive and Liver Disease 2014;46: 27-9. 